\title{
SU UNA MALEDIZIONE OSCA: TEONIMO O MATRONIMICO?
}

\author{
ROMANO LAZZERONI (*)
}

RiASSUNTO. - La formula matronimica in una defixio osca (la cosiddetta "maledizione di Vibia": Vetter, 6) non è, come sostenne Lejeune, un insulto rivolto al personaggio oggetto della maledizione ("figlio di demoniessa" o qualcosa di simile), ma è l'indicazione della filiazione reale. Il matronimico nelle maledizioni è un uso presente in un'area molto vasta, dal Mediterraneo all'India antica. Scopo è l'identificazione inequivoca della persona esecrata.

$$
* * *
$$

ABSTRACT. - The "formula" osca. Maternal filiation or identification of the son?

The formula matronimica in an oscan defixio (the so-called "curse of Vibia": Vetter 6) it is not, as Lejeune sustained, an insult addressed to the subject of the curse ("child of demoniessa" or something similar), but it is the indication of the real filiation. The matronimico in the curses is a use present in a very huge area, from the Mediterranean to the Ancient India. The purpose is the right identification of the execrated person.

In una maledizione capuana (Ve. $\left.{ }^{1}, 6\right)$, la cosiddetta 'maledizione di Vibia', la persona oggetto della defixio è denominata con la filiazione matrilineare: pakiui kluvatiiui valaimas puklui 'A Pacio Klovatio, figlio di Valaima'. La formula è ripetuta sei volte.

Che valaima significhi 'Ottima' non c'è dubbio. Ce lo dice la tavo-

(*) Istituto Lombardo Accademia di Scienze e Lettere, Università degli Studi di Pisa.

1 Con Ve. si indica la silloge di Vetter (Vetter 1953). 
la bantina (Ve., 2, 10): pod valaemom touticom tadait ezom 'quod optimum publicum videatur esse'.

In un saggio del 1968 M. Lejeune scrisse così: "è incontestabile che Valaimas è un genitivo femminile, propriamente quello del superlativo 'Optima' qui usato come nome proprio: non può, dunque, trattarsi di una indicazione normale di filiazione (che sarebbe patronimica). Contesto, di conseguenza, eccezionale. Generalmente in Valaima si vede un nome di donna. Noi, piuttosto, ci vedremo la designazione eufemistica ('optima' per 'pessima') di una divinità infernale. Ogni volta che il suo nome riappare nella lamina, Pacius Clovatius sarebbe qualificato come 'figlio dell'assolutamente buona' cioè di creatura infernale" (1968: 75).

Contro Lejeune scrissi poco dopo (Lazzeroni 1971) sostenendo la tesi che ora ripropongo con qualche aggiunta e modificazione; tesi che riscosse l'approvazione di Rix (1979: 114) e che non meriterebbe di essere riesumata se Lejeune non avesse ribadito la sua nell'Anthroponimie Osque (1976: 43) ripetendo che 'Optima' non è antroponimo femminile e, soprattutto, se Untermann (2000: 822) non avesse definito Valaimas "Vielleicht I(ndividual) N(ame), vielleicht aber auch ein für den Zweck der Verwünschung herangezogener Deckoder Spitzname".

La tesi di Lejeune discende dalla convinzione che in latino puer indichi la filiazione divina e filius quella umana; poiché l'osco pukel avrebbe lo stesso valore di puer, Valaimas dovrebbe essere il genitivo di un teonimo.

A ben vedere, l'argomentazione è circolare: si suppone che pukel indichi, come il latino puer, la filiazione divina e si inferisce che Valaimas sia un teonimo (propriamente il nome tabuizzato di una divinità infernale) perché nella formula onomastica è retto da pukel.

In latino puer designa effettivamente le genealogie divine. Le attestazioni vanno da Cereris Proserpina puer di Nevio ai pueros Ledae di Orazio; ma si tratta sempre di testi poetici, ad eccezione di una iscrizione prenestina (CIL XIV, 2862), che si contrappone ad una più antica in cui compare fileia nell'identico contesto: Fortuna Diovo fileia primogenia.

Non sfuggì a Barchiesi (1962: 428 ss.) che i testi letterari latini (e probabilmente anche l'iscrizione prenestina) calcano modelli greci con

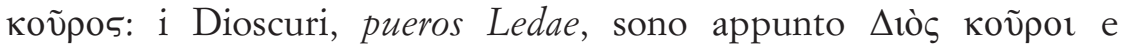

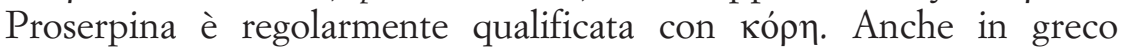




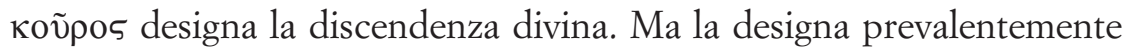

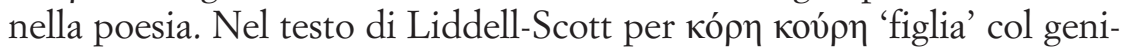
tivo sono citati Omero, Saffo, i Tragici e un solo esempio in una iscrizione tessalica (IG, IX, 2, 1035) e, per кópos кои̃рos, ancora Omero, i Tragici e un comico.

E, dunque, se puer 'figlio' è calcato su ко̃̃pos, si tratta di uno stilema letterario, derivato dalla tradizione epico-tragica greca. Inferire un suo uso istituzionalizzato nella formula onomastica è illegittimo; sarebbe come inferire l'uso istituzionalizzato in qualche tipo di formula onomastica da Gnaivod patre prognatus che si legge sul sepolcro di Scipione Barbato.

A maggior ragione, dal valore che puer assume nella letteratura latina, non si può inferire il valore di pukel in una maledizione osca; tra l'altro, nemmeno sappiamo se pukel si oppone ad un altro nome del figlio, così come puer si oppone a filius. La formula onomastica osca è muta perché il genitivo e più spesso la sigla del nome del padre non sono seguiti dall'appellativo 'figlio': V. Vinikiis $M($ a)r(abeis) ecc. (Ve., 11).

Se l'appellativo 'figlio' compare nella maledizione di Vibia, ciò dipenderà dal fatto che l'indicazione della discendenza materna non appartiene alla formula onomastica ufficiale.

Valaimas 'Optimae' non è un teonimo, ma un nome di donna. Ce lo dice l'onomastica romana: Bonus, Melior, Optimus sono cognomina dei due sessi largamente attestati: Bonus 9 volte al maschile, 17 al femminile; Melior 27 al maschile, 1 al femminile; Optimus/-tumus 10 al maschile, 6 al femminile (Kajanto 1982). Ed è appena il caso di aggiungere che l'uso di designare l'oggetto di una defixio col cognomen è frequente, in particolar modo, ovviamente, quando si tratta di schiavi: Acutus (A.', 218), Chilo (A., 199), Germanus (A., 220), Bonosa (A., 268), Eutychia (A., 138), Rhodine (A., 139) ecc.

Notiamo a questo punto un particolare sfuggito al Lejeune: nelle defixiones greche e latine la formula matrilineare compare con frequenza più che casuale. Il repertorio di Audollent (1904) offre larga messe

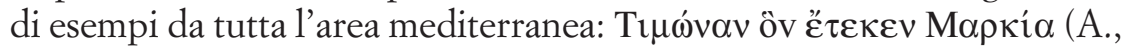
125: Cipro), ( $\Lambda \varepsilon \imath \beta \alpha ́ p ı$ ồ हैं rit Optata (A., 266: Hadrumentum), Maurussum quem peperit Felicitas

2 Con A. si indica la raccolta di defixiones di Audollent (Audollent 1904). 
(A., 250: Cartagine), Lupum qui et vocatur Caucadio qui est filius Sallusties Veneries sive Venerioses (A., 129: Arezzo), ecc. La similitudine con la formula osca non potrebbe essere maggiore.

Quando compare anche un patronimico maschile (A., 198:

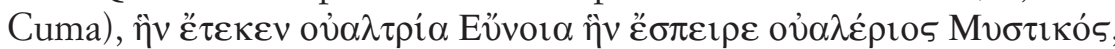
è significativo che il nome della madre preceda quello del padre; se è quello del padre e non piuttosto quello del nonno, padre della madre: $\sigma \pi \varepsilon i \rho \omega$ significa anche 'generare', oltre che 'fecondare'. La madre può ben designarsi col patronimico. Quello che conta è l'identificazione del figlio.

A questo punto l'interpretazione della formula osca è ovvia: Pacio Clovatio è stato designato con la filiazione materna perché la filiazione

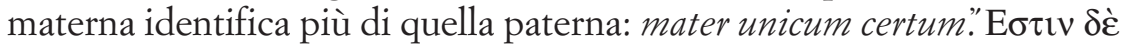

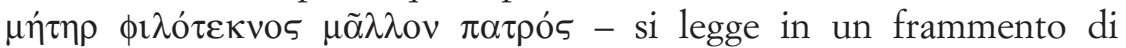

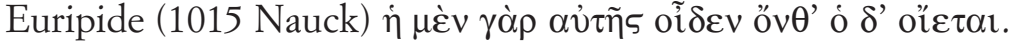

Nella magia nera è indispensabile che l'oggetto di una maledizione sia identificato senza equivoco. Nel mondo indiano la maledizione deve avere l'effetto di 'cancellare l'espirazione e l'ispirazione di un tale, figlio di tal padre e di tal madre' (KS, 47, 12-22); "le formule di imprecazione possono variare all'infinito", scrive Henry di quelle indiane (1904: 224), "ma tutte si riconducono a prototipi che si somigliano e soprattutto comportano sempre una genealogia minuziosa del nemico, per evitare l'errore sulla persona, e ancora di più per assicurare il potere che si ha su questa, per il solo fatto di conoscere il suo nome e quello dei suoi antenati”.

Ecco perché nella maledizione di Vibia la formula onomastica è matrilineare. La formula onomastica, appunto, non l'insulto a un figlio di diavolessa. Di Pacio Clovatio Valaima è la madre naturale.

Resta da concludere. Ciò che ho detto fin qui non è nuovo. Un accenno, purtroppo spesso dimenticato, già si trova nell'introduzione al volume di Audollent sulle defissioni (1904: LI). Ma è importante una considerazione di metodo: se i testi fossero muti la tesi di Lejeune sarebbe sostenibile: in latino puer designa effettivamente la filiazione divina e, considerando quanto l'osco dipenda dal latino nei formulari, non sarebbe assurdo supporre che pukel avesse la stessa funzione. La circolarità dell'argomentazione inviterebbe alla cautela, ma non smentirebbe l'ipotesi. E perché, magari, non pensare anche all'influenza degli Etruschi di Capua, dato che in Etruria la formula matrilineare è frequente nelle iscrizioni sepolcrali? 
Ma i testi parlano e, se li si sa interrogare, ci rivelano le circostanze storiche e culturali sottese alla loro redazione. E ci dicono che puer 'figlio' è un calco dal greco della poesia romana e, verosimilmente, soltanto di questa; e che la filiazione matrilinea della maledizione osca è motivata dalla ideologia sottostante alle defixiones, ideologia documentata in un'area culturale estesissima, dall'India antica al Mediterraneo e, forse, ideologia universale.

Dunque, lasciamo parlare i testi. Cioè sforziamoci di leggere fra le loro righe la storia degli uomini che li hanno creati. 


\section{BIBLIOGRAFIA}

Audollent, A. 1904. Defixionum Tabellae, Parigi, Fontemoing.

Barchiesi, M. 1962. Nevio Epico, Padova, CEDAM.

Henry, V. 1904. La magie dans l'Inde antique, Parigi, Dujarrie \& C.

Kajanto, I. 1982 [1965ํ‥ The Latin Cognomina, Roma, Bretschneider.

Lazzeroni, R. 1971. Contatti di lingue e culture nell'Italia antica: il nome del figlio e quello dei Dioscuri, in "Studi e Saggi Linguistici", 11, pp. 22-47.

Lejeune, M. 1968. 'Fils' et 'fille' dans le langues de l'Italie ancienne, in "Bulletin de la Société de Linguistique de Paris", 62, pp. 67-86.

Lejeune, M. 1968. L'anthroponimie osque, Parigi, Les Belles Lettres.

Rix, H. 1979. Recensione a: Lejeune, M. 1968. L'anthroponimie osque, in "Kratylos", 24, pp. 107-116.

Untermann, J. 2000. Wörterbuch des Oskisch-Umbrischen, Heidelberg, Winter.

Vetter, E. 1953. Handbuch der italischen Dialekte, Heidelberg, Winter. 